Mar. Drugs 2011, 9, 772-789; doi:10.3390/md9050772

Article

\title{
Genetic Approach for the Fast Discovery of Phenazine Producing Bacteria
}

\section{Imke Schneemann, Jutta Wiese, Anna Lena Kunz and Johannes F. Imhoff*}

Kieler Wirkstoff-Zentrum (KiWiZ) am Leibniz-Institut für Meereswissenschaften (IFM-GEOMAR), Am Kiel-Kanal 44, 24106 Kiel, Germany; E-Mails: ischneemann@ifm-geomar.de (I.S.); jwiese@ifm-geomar.de (J.W.); akunz@ifm-geomar.de (A.L.K.)

* Author to whom correspondence should be addressed; E-Mail: jimhoff@ifm-geomar.de; Tel.: +49-431-600-4450; Fax: +49-431-600-4452.

Received: 17 February 2011; in revised form: 1 April 2011 / Accepted: 29 April 2011 / Published: 9 May 2011

\begin{abstract}
A fast and efficient approach was established to identify bacteria possessing the potential to biosynthesize phenazines, which are of special interest regarding their antimicrobial activities. Sequences of $p h z \mathrm{E}$ genes, which are part of the phenazine biosynthetic pathway, were used to design one universal primer system and to analyze the ability of bacteria to produce phenazine. Diverse bacteria from different marine habitats and belonging to six major phylogenetic lines were investigated. Bacteria exhibiting $p h z \mathrm{E}$ gene fragments affiliated to Firmicutes, Alpha- and Gammaproteobacteria, and Actinobacteria. Thus, these are the first primers for amplifying gene fragments from Firmicutes and Alphaproteobacteria. The genetic potential for phenazine production was shown for four type strains belonging to the genera Streptomyces and Pseudomonas as well as for 13 environmental isolates from marine habitats. For the first time, the genetic ability of phenazine biosynthesis was verified by analyzing the metabolite pattern of all PCR-positive strains via HPLC-UV/MS. Phenazine production was demonstrated for the type strains known to produce endophenazines, 2-hydroxy-phenazine, phenazine-1-carboxylic acid, phenazine-1,6-dicarboxylic acid, and chlororaphin as well as for members of marine Actinobacteria. Interestingly, a number of unidentified phenazines possibly represent new phenazine structures.
\end{abstract}

Keywords: phenazine; Actinobacteria; oligonucleotides; HPLC-UV/MS 


\section{Introduction}

Nature is a profitable source of pharmaceutically active substances covering the whole range of biological activities such as antimicrobial, antitumoral, antiparasitic or immunosuppressive [1,2]. Various natural products or natural product-derived compounds have been approved as drugs or are undergoing clinical evaluation and registration [2,3]. Because the rediscovery rate of already known substances in standard screening approaches is high, new strategies are urgently needed. Genetic approaches for the detection of secondary metabolite pathways are promising tools for the selection of biosynthetically talented microorganisms. So far, main targets in this respect were genes encoding for polyketide synthases (PKSs) or non-ribosomal peptide synthetases (NRPSs) [4,5]. By focusing on polyketides and non-ribosomal peptides other interesting compound classes such as phenazines have been neglected so far. Phenazines of natural or synthetic origin turned out to be good drug candidates and therefore are promising secondary metabolites [6]. They are heterocyclic, nitrogenous compounds that are substituted at different sites of the core ring system and therefore display a wide range of structural derivatives and biological activities. More than 100 biologically active (antibacterial, antifungal, antiviral, antitumor) phenazines from natural origin are known to date, synthesized mainly by Pseudomonas and Streptomyces species [7]. Phenazines produced by the root-colonizing Pseudomonas fluorescens 2-79 and Pseudomonas aureofaciens 30-84 are good examples for congeners with promising biological activity, in this case inhibiting several fungal plant pathogens [8]. An economically successful phenazine is clofazimine (Lamprene ${ }^{\circledR}$, Novartis), first described in 1957 which was established as an antileprosy agent and exerts anti-inflammatory properties in controlling erythema nodosum leprosum reactions (Official FDA Drug label) [6] as well as other antimicrobial activities [9-11]. Clofazimine is a synthetic phenazine analogue belonging to the riminophenazines, a group of compounds which was originally discovered in lichens [12,13]. Another phenazine, bis(phenazine-1-carboxamide), acts as a potent cytotoxin and represents an interesting class of dual topoisomerase I/II directed anticancer drugs [14].

Although principal parts of the phenazine biosynthesis (Figure 1) and their genetics are known [15,16], there are still gaps in our knowledge and the phenazine pathway is still hypothetical [17]. The function and gene products of $p h z \mathrm{C}, p h z \mathrm{E}$ and $p h z \mathrm{D}$ have been experimentally proven. These enzymes catalyze the conversion into 3-deoxy-D-arabino-heptulosonic acid 7-phosphate (DAHP), 2-amino-2-deoxyisochorismic acid (ADIC) and trans-2,3-dihydro-3-hydroxyanthranilic acid (DHHA) [17-20]. The phenazine core structure is synthesized subsequently by condensation of two molecules of 6-amino-5-oxocyclohex-2-ene-1-carboxylic acid [17]. Presumably, PhzF is responsible for the isomerization of DHHA [18]. By now, the function of the enzymes $P h z \mathrm{~A}, P h z \mathrm{~B}$ and $P h z \mathrm{G}$ are almost enlightened [19] and their role in the condensation and rearrangement reactions to form PCA is demonstrated [17]. According to this information, the design of appropriate oligonucleotide probes and primers was possible. Mavrodi et al. (2010) [21] quite recently designed four different pairs of oligonucleotides on the basis of the $p h z \mathrm{~F}$ gene of the phenazine biosynthesis and investigated the diversity of phenazine producers mainly in soil-dwelling and plant-associated bacteria.

In particular marine bacteria have attracted our interest to search for promising natural products [1,22-26]. Therefore, we have investigated the possible application of a genetic approach for the detection of new marine isolates using $p h z \mathrm{E}$ gene fragments as markers for the ability to produce 
bioactive phenazines. In contrast to Mavrodi et al. (2010) [21], we wanted to design a more universal primer system able to study a wide range of bacterial groups including actinomycetes and pseudomonades, in order to detect genes of phenazine production in unidentified new isolates without prior performance of a phylogenetic classification.

Figure 1. Schematic representation of the phenazine biosynthesis pathway.

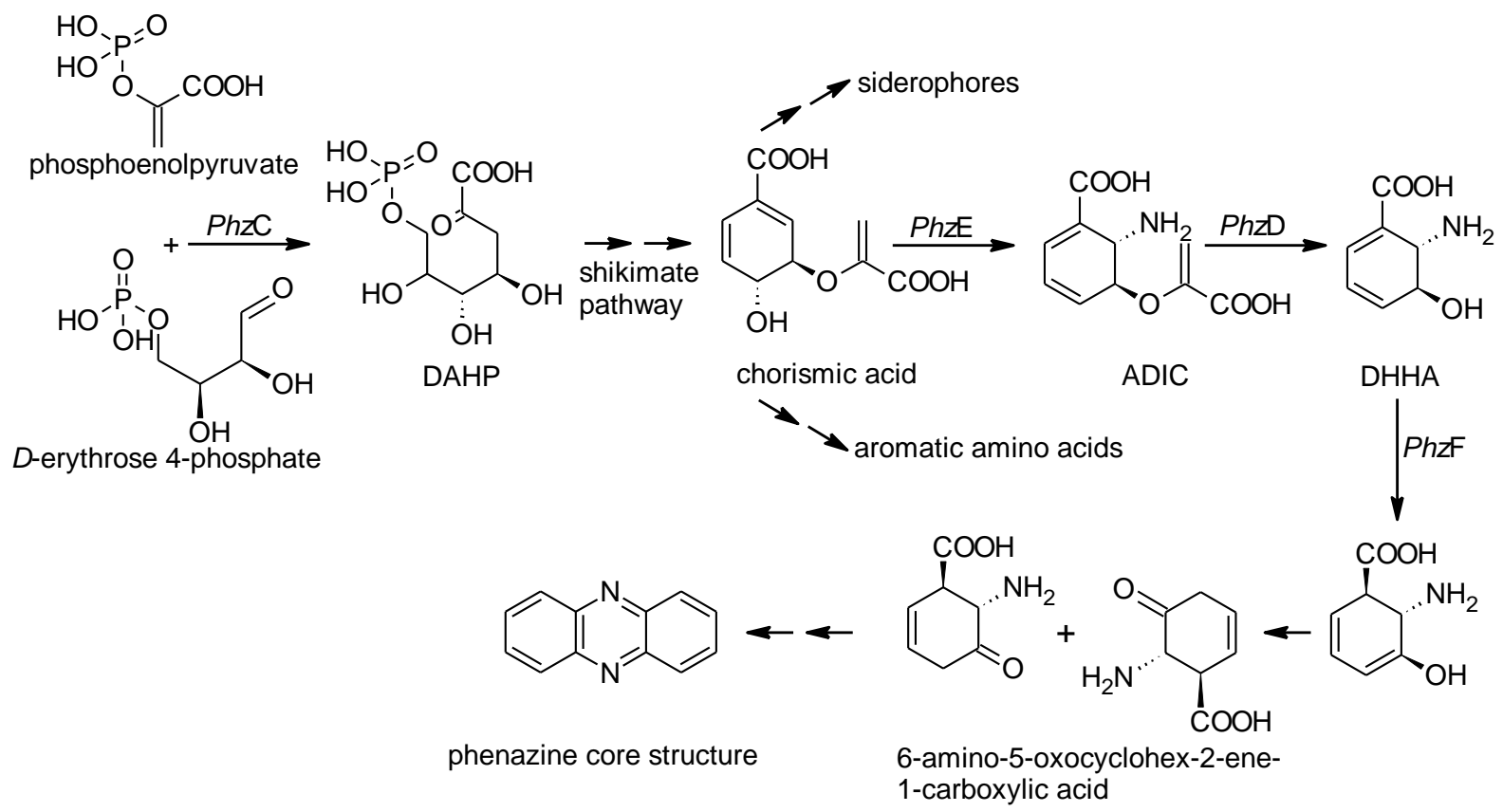

\section{Results and Discussion}

The high diversity of microbes and their manifold biosynthetic capabilities offer a great potential for novel, bioactive secondary products. Genetic approaches such as the application of primer systems for NRPS, PKS or halogenases are efficient methods to select appropriate strains for further analyses [27,28]. The screening of bacteria for the presence of phenazine genes significantly adds to these selection filters. We report here on a rapid universal genetic system for the discovery of bacteria that are able to produce phenazines. The suitability of this PCR based screening approach for the identification of phenazine producing bacteria was demonstrated. A gene fragment indispensable for phenazine biosynthesis $(p h z \mathrm{E})$ served as a template for primer generation. As positive controls the type strain of S. cinnamonensis, and three subspecies of Pseudomonas chlororaphis, which are all well known producers of phenazines, were included in this study. For control strains several phenazine biosynthesis gene sequences were available at the NCBI (e.g., AM384985, HM594285, AF007801). Altogether 168 bacterial strains (including the four reference organisms) were examined for the presence of $p h z \mathrm{E}$. The PCR analysis was complemented by a chemical investigation in order to demonstrate the expression of the biosynthesis genes and to confirm the production of phenazines as proof of concept. 


\subsection{Design of Oligonucleotides to Search for phzE Phenazine Gene Fragments}

Former investigations were able to associate different steps of the phenazine biosynthetic pathway with the corresponding genes, e.g., $p h z \mathrm{C}, p h z \mathrm{D}, p h z \mathrm{E}$ and $p h z \mathrm{~F}$. The transformation from chorismate to 2-amino-2-deoxyisochorismic acid (ADIC) is necessary for the formation of the core structure of phenazines and is catalyzed by the enzyme $P h z \mathrm{E}$. Thus, $P h z \mathrm{E}$ is a key enzyme in phenazine biosynthesis and the corresponding gene $p h z \mathrm{E}$ is suitable for primer design. Sequences from the phenazine biosynthetic pathway for Alpha-, Beta- and Gammaproteobacteria, Actinomycetes and Firmicutes are available at the homepage of the National Centre for Biotechnology Information (NCBI) and known from literature [29,30]. To ensure the inclusion of only true phenazine sequences, oligonucleotide primers were constructed only from those genes known to be involved in the biosynthesis of corresponding chemical substances. Two conserved sites occurred within the alignment of $p h z \mathrm{E}$ sequences (Figure 2), which had a distance to each other to produce fragments of an appropriate length. The degenerated primers $p h z \mathrm{Ef}$ (5'-GAA GGC GCC AAC TTC GTY ATC AA-3') and phzEr (5'-GCC YTC GAT GAA GTA CTC GGT GTG-3') were designed to amplify a highly conserved stretch of the $p h z \mathrm{E}$ gene of approximately $450 \mathrm{bp}$. The comparison of the oligonucleotide sequences from designed $p h z \mathrm{Ef}$ and $p h z \mathrm{Er}$ primers with known phenazine genes verified this stretch as highly specific for $p h z \mathrm{E}$ genes. Because the basic phenazine gene cluster including the $p h z \mathrm{E}$ gene is highly conserved and derivatization of the basic phenazine structure are made at a later stage in the biosynthesis, the constructed $p h z \mathrm{E}$ primers are expected to detect genes of a large variety of different phenazine structures and are appropriate to search for unknown bacteria producing novel phenazines.

Figure 2. Alignment of known phzE gene sequences. Marked blocks served as the basis for primer construction.

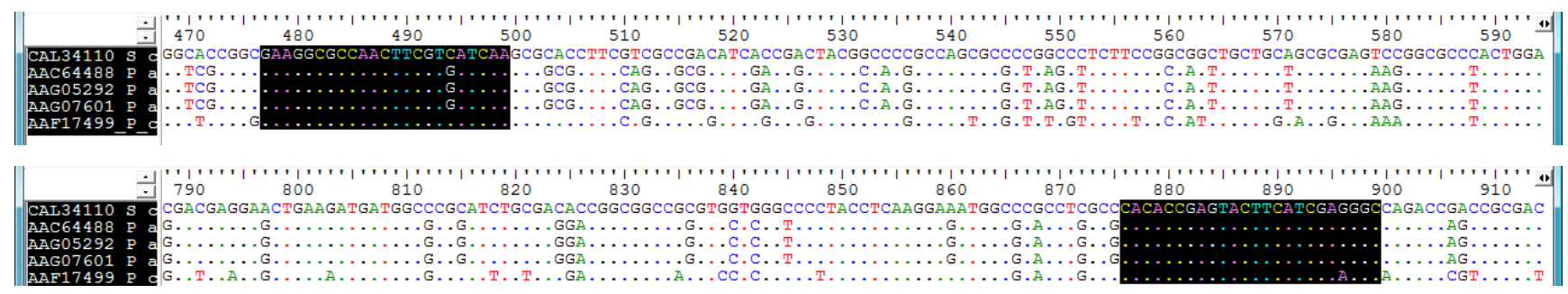

\subsection{Screening for phzE Gene Fragments with the Constructed Primers}

Genes belonging to the phenazine biosynthetic pathway were present in approximately $10 \%$ of the bacterial strains analyzed. PCR results of 13 (8\%) out of 164 bacterial strains and four reference organisms were positive in regard to the presence of $p h z \mathrm{E}$ gene fragments (Figure 3, Tables 1 and 2). The investigated bacteria comprised different bacterial phyla, namely Actinobacteria (76), Bacteroidetes (2), Firmicutes (28) and Proteobacteria (62) (Table 1). 
Figure 3. $P h z \mathrm{E}$ gene fragment amplification of selected strains. Lane 1 and 10: $1 \mathrm{~kb}$ DNA-ladder; 2: positive control Pseudomonas chlororaphis subsp. chlororaphis DSM $50083^{\mathrm{T}}$; 3: negative control (without template DNA); 4, 5, and 6: isolates H253, HB117, and LB129, respectively, exhibiting phzE fragments; 7, 8, and 9: isolates HB290, HB147, and LB164, respectively, exhibiting nor $p h z \mathrm{E}$ fragments.



Table 1. Distribution of $p h z \mathrm{E}$ phenazine genes among major phylogenetic groups and phenazine production of cultures (number of strains).

\begin{tabular}{lccc}
\hline \multirow{2}{*}{ Phylogenetic group } & \multicolumn{3}{c}{ Number of strains } \\
\cline { 2 - 4 } & Analyzed & $\begin{array}{c}\text { PCR amplification } \\
\text { phzE gene positive }\end{array}$ & $\begin{array}{c}\text { Producing phenazines } \\
\text { in culture }\end{array}$ \\
\hline Actinobacteria $^{\mathrm{a}}$ & 76 & 11 & 11 \\
Bacteroidetes $_{\text {Firmicutes }}$ & 2 & 0 & 0 \\
Alphaproteobacteria & 28 & 1 & 0 \\
Betaproteobacteria $_{\text {Gammaproteobacteria }}^{\mathrm{b}}$ & 21 & 2 & 0 \\
In total & 2 & 0 & 0 \\
\hline
\end{tabular}

${ }^{a}$ including control strain $S$. cinnamonensis.

${ }^{\mathrm{b}}$ including 3 control P. chlororaphis strains.

Corresponding gene fragments were detected in 11 strains of Actinobacteria, one strain of Firmicutes and two strains of the Alphaproteobacteria. All sequences were similar to known phzE gene sequences in a range from $65 \%$ similarity ( $p h z \mathrm{E}$ of strain LB151 to phzE of $P$. chlororaphis, $\mathrm{AAF} 17499$ ) to $95 \%$ similarity ( $p h z \mathrm{E}$ of strain $\mathrm{AB} 108$ to $p h z \mathrm{E}$ of gene from $S$. cinnamonensis, CAL34110) (Table 2). Regarding the environmental isolates none of the strains within the Bacteroidetes, Beta- as well as Gammaproteobacteria could be shown to contain phzE in PCR amplification. This was unexpected, because among the 36 gammaproteobacterial isolates 18 Pseudomonas strains were examined and our PCR approach was performed with primer sequences largely based on sequences from Pseudomonas strains known as producers of phenazines [16,31,32]. The suitability of our primer set to detect phenazine genes in Pseudomonas species was further demonstrated by performing a database search that matched perfectly several phenazine genes, e.g., 
P. chlororaphis (L48339), Pseudomonas sp. M18 (FJ494909), P. aeruginosa (FM209186, CP000744, CP000438, AE004091, AF005404). Anyhow, a study based on $p h z F$ sequences exhibited a hit ratio of 100\% including 51 Pseudomonas strains [21]. Therefore, all 18 pseudomonads from our study exhibiting negative results using $p h z \mathrm{E}$ primers were subjected to a genetic approach with $p h z \mathrm{~F}$ primers. While the PCR-amplification of $p h z \mathrm{E}$ and $p h z \mathrm{~F}$ gene fragments of the control type strains was positive (Table 2), amplification of the investigated isolates failed. As an additional control experiment, crude extracts of six Pseudomonas strains were analyzed by HPLC-UV/MS. Because of the distinctive properties of phenazine UV-absorption spectra the presence of phenazine metabolites was out of question. Additionally, for another study all natural products from two of the investigated Pseudomonas strains were isolated and chemically identified. No phenazines were detected. We conclude that the Pseudomonas strains analyzed in this study lack genes for phenazine production and are unable to produce phenazines. In agreement with this, the only known marine phenazine producing Pseudomonas species is $P$. aeruginosa [33-35], synthesizing almost always pyocyanin. In contrast, different marine streptomycetes are known for production of variable phenazine structures [9]. Streptomyces strains in this study are the most productive group as well. While Brevibacterium, Bacillus and Pelagibacter were known as marine phenazine producers [36-38], this is the first time that representatives of the genera Micromonospora, Kiloniella and Pseudovibrio were identified as marine phenazine producers as well.

Table 2. Presence of phenazine biosynthesis genes in the strains investigated. Information on related type strains (according to $16 \mathrm{~S}$ rRNA sequences) and sequence similarity of phzE genes to known phenazine genes is displayed. Length, similarity and original bearer of the genetic information of the $p h z \mathrm{E}$ PCR products are also included.

\begin{tabular}{|c|c|c|c|c|}
\hline Strain no. & $\begin{array}{l}\text { Next relative type strain } \\
\text { and acc.-no. }{ }^{a}\end{array}$ & Phylum $^{b}$ & $\begin{array}{l}\text { Sequence } \\
\text { length }^{c}\end{array}$ & $\begin{array}{l}\text { Related phenazine } \\
\text { gene, acc.-no. }{ }^{d} \text {, } \\
\text { similarity } \\
\text { and producer }\end{array}$ \\
\hline Strains used as & Positive control & & & \\
\hline DSM $1042^{\mathrm{T}}$ & $\begin{array}{l}\text { Streptomyces cinnamonensis DSM } \\
1042^{\mathrm{T}} ; \mathrm{DQ} 462657\end{array}$ & A & 127 & $\begin{array}{l}\text { phzE; CAL34110; } 100 \% \text {; } \\
\text { S. cinnamonenis }\end{array}$ \\
\hline DSM $6698^{\mathrm{T}}$ & $\begin{array}{l}\text { Pseudomonas chlororaphis subsp. } \\
\text { aureofaciens DSM } 6698^{\mathrm{T}} \text {; } \\
\text { AY509898 }\end{array}$ & GP & 139 & $\begin{array}{l}\text { phzE: ADP } 21173 ; 100 \% \\
\text { P. chlororaphis } \\
\text { phzF: ADP21174; } 100 \% \\
\text { P. chlororaphis }\end{array}$ \\
\hline DSM $19603^{\mathrm{T}}$ & $\begin{array}{l}\text { Pseudomonas chlororaphis subsp. } \\
\text { aurantiaca DSM } 19603^{\mathrm{T}} \text {; DQ682655 }\end{array}$ & GP & 137 & $\begin{array}{l}\text { phzE: ADP } 21173 ; 98 \% \\
\text { P. chlororaphis } \\
\text { phzF: ADP } 2117449 \% \\
\text { P. chlororaphis }\end{array}$ \\
\hline DSM $50083^{\mathrm{T}}$ & $\begin{array}{l}\text { Pseudomonas chlororaphis subsp. } \\
\text { chlororaphis DSM } 50083^{\mathrm{T}} ; \mathrm{Z76673}\end{array}$ & GP & 125 & $\begin{array}{l}\text { phzE; AAF } 17499 ; 92 \% \\
\text { P. chlororaphis } \\
\text { phzF: AAF } 17500 ; 99 \% \\
\text { P. chlororaphis }\end{array}$ \\
\hline
\end{tabular}


Table 2. Cont.

\begin{tabular}{|c|c|c|c|c|}
\hline Strain no. & $\begin{array}{l}\text { Next relative type strain } \\
\text { and acc.-no. }{ }^{a}\end{array}$ & Phylum $^{b}$ & $\begin{array}{l}\text { Sequence } \\
\text { length }^{c}\end{array}$ & $\begin{array}{l}\text { Related phenazine } \\
\text { gene, acc.-no. }{ }^{d} \text {, } \\
\text { similarity } \\
\text { and producer }\end{array}$ \\
\hline Environmental & Isolates & & & \\
\hline AB 108 & $\begin{array}{l}\text { Pseudovibrio ascidiaceicola } \mathrm{F} 423^{\mathrm{T}} \\
\mathrm{AB} 175663\end{array}$ & $\mathrm{AP}$ & 144 & $\begin{array}{l}\text { phzE; CAL34110; 95\%; } \\
\text { S. cinnamonenis }\end{array}$ \\
\hline HB117 & $\begin{array}{l}\text { Streptomyces fulvorobeus LMG } \\
\text { 19901 }^{\mathrm{T}} \text {; AJ781331 }\end{array}$ & A & 141 & $\begin{array}{l}\text { phzE; AAF } 17499 ; 73 \% ; \\
\text { P. chlororaphis }\end{array}$ \\
\hline HB 122 & $\begin{array}{l}\text { Streptomyces luridiscabiei } \mathrm{S} 63^{\mathrm{T}} \\
\text { AF361784 }\end{array}$ & A & 141 & $\begin{array}{l}\text { phzE; AAF } 17499 ; 74 \% ; \\
\text { P. chlororaphis }\end{array}$ \\
\hline HB202 & $\begin{array}{l}\text { Streptomyces mediolani LMG } \\
20093^{\mathrm{T}} ; \text { AJ781354 }\end{array}$ & A & 91 & $\begin{array}{l}\text { phzE; NP_252903; 84\%; } \\
\text { P. aeruginosa }\end{array}$ \\
\hline HB253 & $\begin{array}{l}\text { Micromonospora matsumotoense } \\
\text { IMSNU } 22003^{\mathrm{T}} \text {; AF152109 }\end{array}$ & A & 144 & $\begin{array}{l}\text { phzB; AAF17496; 67\%; } \\
\text { P. chlororaphis }\end{array}$ \\
\hline HB254 & $\begin{array}{l}\text { Micromonospora matsumotoense } \\
\text { IMSNU } 22003^{\mathrm{T}} \text {; AF } 152109\end{array}$ & A & 140 & $\begin{array}{l}\text { phzE; AAF } 17499 ; 73 \% ; \\
\text { P. chlororaphis }\end{array}$ \\
\hline HB291 & $\begin{array}{l}\text { Streptomyces fulvorobeus LMG } \\
\text { 19901 }^{\mathrm{T}} \text {; AJ781331 }\end{array}$ & A & 140 & $\begin{array}{l}\text { phzE; AAF } 17499 ; 73 \% ; \\
\text { P. chlororaphis }\end{array}$ \\
\hline LB066 & $\begin{array}{l}\text { Kiloniella laminariae } \mathrm{LD}^{\mathrm{T}}{ }^{\mathrm{T}} \text {; } \\
\text { AM749667 }\end{array}$ & $\mathrm{AL}$ & 132 & $\begin{array}{l}\text { phzE; CAL34110; } 91 \% \text {; } \\
\text { S. cinnamonenis }\end{array}$ \\
\hline LB114 & $\begin{array}{l}\text { Streptomyces flavogriseus DSM } \\
40323^{\mathrm{T}} \text {; AJ494864 }\end{array}$ & A & 141 & $\begin{array}{l}\text { phzE; AAF } 17499 ; 79 \% \text {; } \\
\text { P. chlororaphis }\end{array}$ \\
\hline LB129 & $\begin{array}{l}\text { Streptomyces fimicarius ISP } 5322^{\mathrm{T}} \text {; } \\
\text { AY } 999784\end{array}$ & A & 145 & $\begin{array}{l}\text { phzB; AAF } 17496 ; 75 \% ; \\
\text { P. chlororaphis }\end{array}$ \\
\hline LB150 & $\begin{array}{l}\text { Streptomyces luridiscabiei } \mathrm{S} 63^{\mathrm{T}} \text {; } \\
\text { AF361784 }\end{array}$ & A & 132 & $\begin{array}{l}\text { phzB; AAF } 17496 ; 74 \% ; \\
\text { P. chlororaphis }\end{array}$ \\
\hline LB151 & $\begin{array}{l}\text { Streptomyces griseus ATCC } 51928^{\mathrm{T}} \text {; } \\
\text { AF112160 }\end{array}$ & A & 133 & $\begin{array}{l}\text { phzE; AAF } 17499 ; 65 \% \text {; } \\
\text { P. chlororaphis }\end{array}$ \\
\hline
\end{tabular}

\footnotetext{
${ }^{a} \mathrm{NCBI}$ accession number.

(all sequences were at least $98.5 \%$ similar to the corresponding type strain).

${ }^{b} \mathrm{~A}=$ Actinobacteria, $\mathrm{AP}=$ Alphaproteobacteria, $\mathrm{GP}=$ Gammaproteobacteria, $\mathrm{F}=$ Firmicutes.

${ }^{c}$ Given is the number of amino acids.

${ }^{d} \mathrm{NCBI}$ accession number.
}

\subsection{Detection of Phenazines in the phzE Positive Strains}

To demonstrate the synthesis of phenazines in all $p h z \mathrm{E}$ positive strains, cultures of these strains were extracted and analyzed by HPLC-UV/MS analyses. 14 out of 17 of these strains were able to produce one or more substances with molecular masses and UV-spectra similar to known phenazines (Table 3, Figure 4a-c). In S. cinnamonensis DSM $1042^{\mathrm{T}}$ the production of endophenazines A-C (Figure 5) and phenazine-1,6-dicarboxylic acid [15] could be demonstrated (Figure 3a). The metabolite chlororaphin was discovered from Pseudomonas chlororaphis subsp. chlororaphis DSM 50083 $3^{\mathrm{T}}$. 2-hydroxy-phenazine (Figure 5) and phenazine-1-carboxylic acid were produced by Pseudomonas chlororaphis subsp. aureofaciens DSM $6698^{\mathrm{T}}$ and Pseudomonas chlororaphis subsp. aurantiaca DSM 
$19603^{\mathrm{T}}$. In addition, the presence of senacarcin A (strain Streptomyces sp. HB117), saphenyl ester D, aestivophoenin C and a derivative thereof (strains Streptomyces sp. HB122 and HB291) as well as phencomycin methyl ester and 1-carboxymethyl phenazine from strain Streptomyces sp. LB129 (Figure 3b) were identified.

All environmental isolates producing phenazines (6\%) were marine Streptomyces sp. or Micromonospora sp. strains. Most of these strains produced both known phenazines and phenazines which did not show any accordance to a database entry. In total, 22 known phenazines were identified. In the case of strain Streptomyces sp. HB202 (Figure 4c), the production of streptophenazines A-H was verified using NMR spectroscopic analyses [39]. The large number of Streptomyces strains containing $p h z \mathrm{E}$ genes is in good agreement with previous reports describing streptomycetes as a rich source for phenazines $[9,15,39,40]$.

Table 3. Known and putative novel phenazines from the strains studied.

\begin{tabular}{|c|c|c|c|c|}
\hline Strain no. & $\begin{array}{l}\text { Next relative type } \\
\text { strain }\end{array}$ & {$\left[\mathbf{M}^{+}\right]$} & $\begin{array}{l}\text { UV absorption } \\
{\text { maxima }(\mathbf{n m})^{a}}\end{array}$ & Dereplication of phenazines \\
\hline Strains used as & positive control & & & \\
\hline \multirow[t]{8}{*}{ DSM $1042^{\mathrm{T}}$} & Streptomyces & 206 & $327,249,212$ & no hit in database \\
\hline & cinnamonensis DSM $1042^{\mathrm{T}}$ & 224 & $371,249,215$ & phenazine-1-carboxylic acid [41] \\
\hline & & 268 & $375,256,223$ & phenazine-1,6-dicarboxylic acid [15] \\
\hline & & 292 & $371,254,214$ & endophenazine $\mathrm{A}[15]^{c}$ \\
\hline & & 306 & $387,269,211$ & no hit in database \\
\hline & & 308 & $372,249,212$ & endophenazine C [15] \\
\hline & & 322 & $375,256,223$ & endophenazine $\mathrm{B}[15]^{c}$ \\
\hline & & 336 & $372,249,212$ & no hit in database \\
\hline \multirow[t]{2}{*}{ DSM $6698^{\mathrm{T}}$} & Pseudomonas chlororaphis & 196 & $368,257,219$ & 2-hydroxy-phenazine [42] \\
\hline & $\begin{array}{l}\text { subsp. aureofaciens DSM } \\
6698^{\mathrm{T}}\end{array}$ & 224 & $371,249,215$ & phenazine-1-carboxylic acid [41] \\
\hline \multirow[t]{2}{*}{ DSM $19603^{T}$} & Pseudomonas chlororaphis & 196 & $368,257,219$ & 2-hydroxy-phenazine $[42]^{c}$ \\
\hline & $\begin{array}{l}\text { subsp. aurantiaca DSM } \\
19603^{\mathrm{T}}\end{array}$ & 224 & $371,249,215$ & phenazine-1-carboxylic acid $[41]^{\mathrm{c}}$ \\
\hline $\operatorname{DSM} 50083^{\mathrm{T}}$ & $\begin{array}{l}\text { Pseudomonas chlororaphis } \\
\text { subsp. chlororaphis DSM } \\
50083^{\mathrm{T}}\end{array}$ & 223 & $370,248,213$ & chlororaphin [43] \\
\hline Environmental & Isolates & & & \\
\hline \multirow[t]{2}{*}{ HB117 } & Streptomyces fulvorobeus & 494 & $370($ br), 274,224 & Senacarcin A \\
\hline & LMG $19901^{\mathrm{T}}$ & 512 & $370(\mathrm{br}), 275,230$ & saphenyl ester D [29] \\
\hline \multirow[t]{5}{*}{ HB122 } & Streptomyces luridiscabiei & 492 & $376,275,235 \mathrm{sh}$ & saphenyl ester D [29] \\
\hline & $\mathrm{S} 63^{\mathrm{T}}$ & 496 & $\begin{array}{l}438 \mathrm{sh}, 383(\mathrm{br}), 276 \\
227\end{array}$ & no hit in database \\
\hline & & 498 & $\begin{array}{l}419 \mathrm{sh}, 393-325,289 \\
253 \mathrm{sh}, 220\end{array}$ & no hit in database \\
\hline & & 508 & $376,275,235 \mathrm{sh}$ & no hit in database \\
\hline & & 510 & $430(\mathrm{br}), 325,224$ & derivative of aestivophoenin C [44] \\
\hline
\end{tabular}


Table 3. Cont.

\begin{tabular}{|c|c|c|c|c|}
\hline Strain no. & $\begin{array}{l}\text { Next relative type } \\
\text { strain }\end{array}$ & {$\left[\mathbf{M}^{+}\right]$} & $\begin{array}{l}\text { UV absorption } \\
{\text { maxima }(\mathbf{n m})^{a}}\end{array}$ & Dereplication of phenazines \\
\hline & & 512 & 432(br), 327, 226 & aestivophoenin C [44] \\
\hline \multirow[t]{8}{*}{ HB202 } & 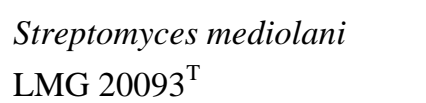 & 396 & $\begin{array}{l}368,364 \text { sh, } 351 \text { sh, } 252, \\
218\end{array}$ & streptophenazines E [39] ${ }^{c}$ \\
\hline & & 410 & $\begin{array}{l}371,364 \text { sh, 354sh, 252, } \\
213\end{array}$ & streptophenazines C [39] \\
\hline & & 410 & $\begin{array}{l}368,364 \text { sh, } 351 \text { sh. } 252, \\
218\end{array}$ & streptophenazines D [39] ${ }^{c}$ \\
\hline & & 424 & $\begin{array}{l}367,363 \mathrm{sh}, 350 \mathrm{sh}, 252, \\
215\end{array}$ & streptophenazines A [39] \\
\hline & & 424 & $\begin{array}{l}368,364 \text { sh, } 351 \text { sh, } 252, \\
218\end{array}$ & streptophenazines B [39 $]^{c}$ \\
\hline & & 438 & $\begin{array}{l}368,364 \mathrm{sh}, 353 \mathrm{sh}, 252, \\
215\end{array}$ & streptophenazines $\mathrm{F}[39]^{c}$ \\
\hline & & 438 & $\begin{array}{l}368,363 \mathrm{sh}, 351 \mathrm{sh}, 252, \\
214\end{array}$ & streptophenazines G [39] \\
\hline & & 440 & $\begin{array}{l}368,363 \mathrm{sh}, 352 \mathrm{sh}, 252, \\
215\end{array}$ & streptophenazines H [39] \\
\hline \multirow[t]{3}{*}{ HB253 } & Micromonospora & 260 & $458,302 \mathrm{sh}, 261,232$ & no hit in database \\
\hline & matsumotoense IMSNU & 465 & $362 \mathrm{sh}, 345,299,221$ & no hit in database \\
\hline & $22003^{\mathrm{T}}$ & 566 & $362 \mathrm{sh}, 345,299,221$ & no hit in database \\
\hline HB254 & $\begin{array}{l}\text { Micromonospora } \\
\text { matsumotoense IMSNU } \\
22003^{\mathrm{T}}\end{array}$ & 451 & $361,343,352,301,223$ & no hit in database \\
\hline \multirow[t]{6}{*}{ HB291 } & Streptomyces fulvorobeus & 492 & $376,275,235 \mathrm{sh}$ & saphenyl ester D [29] \\
\hline & LMG $19901^{\mathrm{T}}$ & 496 & $\begin{array}{l}438 \text { sh, 383(br), 276, } \\
227\end{array}$ & no hit in database \\
\hline & & 498 & $\begin{array}{l}419 \mathrm{sh}, 393-325,289, \\
253 \mathrm{sh}, 220\end{array}$ & no hit in database \\
\hline & & 508 & $376,275,235 \mathrm{sh}$ & no hit in database \\
\hline & & 510 & $430(\mathrm{br}), 325,224$ & derivative of aestivophoenin C [44] \\
\hline & & 512 & 432(br), 327, 226 & aestivophoenin C [44] \\
\hline LB114 & Streptomyces flavogriseus & n.d. ${ }^{b}$ & $370,270,244$ & no hit in database \\
\hline & DSM $40323^{\mathrm{T}}$ & n.d. ${ }^{b}$ & $419,367,305,228$ & no hit in database \\
\hline LB129 & Streptomyces fimicarius ISP & 296 & $366,249,214$ & phencomycin methyl ester [9] \\
\hline & $5322^{\mathrm{T}}$ & 238 & $366,249,214$ & 1-carboxymethyl phenazine \\
\hline LB150 & $\begin{array}{l}\text { Streptomyces luridiscabiei } \\
\mathrm{S} 63^{\mathrm{T}}\end{array}$ & 510 & $\operatorname{sh} 401,378,274,227$ & no hit in database \\
\hline LB151 & $\begin{array}{l}\text { Streptomyces griseus } \\
\text { ATCC } 51928^{\mathrm{T}}\end{array}$ & 510 & $\operatorname{sh} 401,378,274,227$ & no hit in database \\
\hline
\end{tabular}

${ }^{a}$ sh, shoulder, br, broad.

${ }^{b}$ n.d., not detectable.

${ }^{c}$ substance was isolated by prepHPLC and structure was identified by UV-MS and ${ }^{1} \mathrm{H}$ NMR analysis (data not shown). 
Figure 4. (a) UV/MS-chromatogram (black line: MS; green line: UV at $250 \mathrm{~nm}$ ) of a 17 day-old GYM agar plate of strain Streptomyces cinnamonensis DSM $1042^{\mathrm{T}}$. Endophenazine A-C, phenazine-1-carboxylic acid, phenazine-1,6-dicarboxylic acid and an unknown phenazine substance were detected as well as naphterpin and furanonaphthachinon I; (b) UV/MS-chromatogram (black line: MS; green line: UV at $250 \mathrm{~nm}$ ) of a 17 day-old GYM agar plate of Streptomyces strain LB129. 1-carboxymethyl phenazine and phencomycin methyl ester were detected; (c) UV/MS-chromatogram (black line: MS; green line: UV at $250 \mathrm{~nm}$ ) of a 17 day-old GYM agar plate of strain HB202. Different streptophenazines and the aromatic polyketide mayamycin were detected.
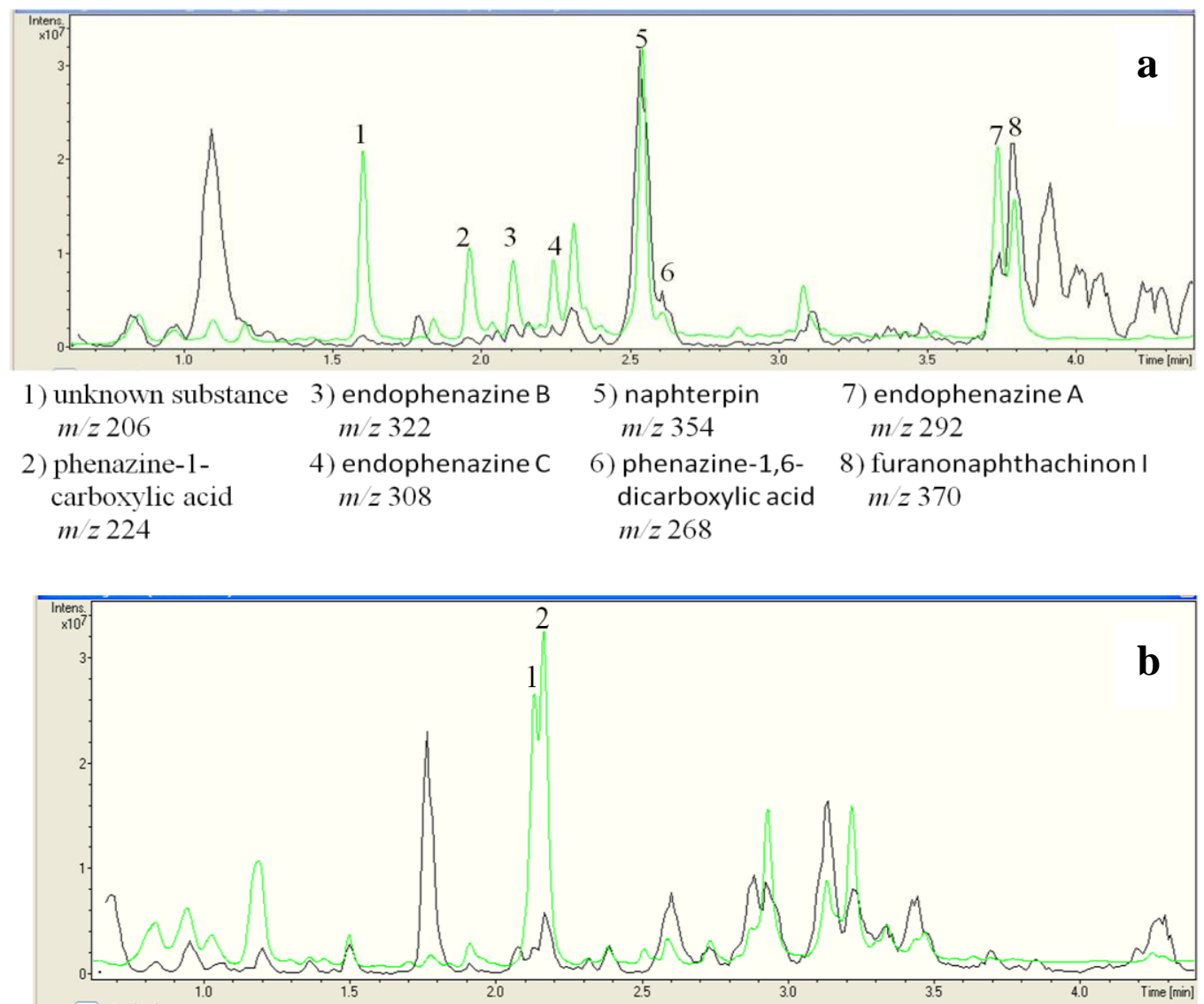
1) 1-carboxymethyl phenazine
2) phencomycin methyl ester $m / z 238$ $m / z 296$

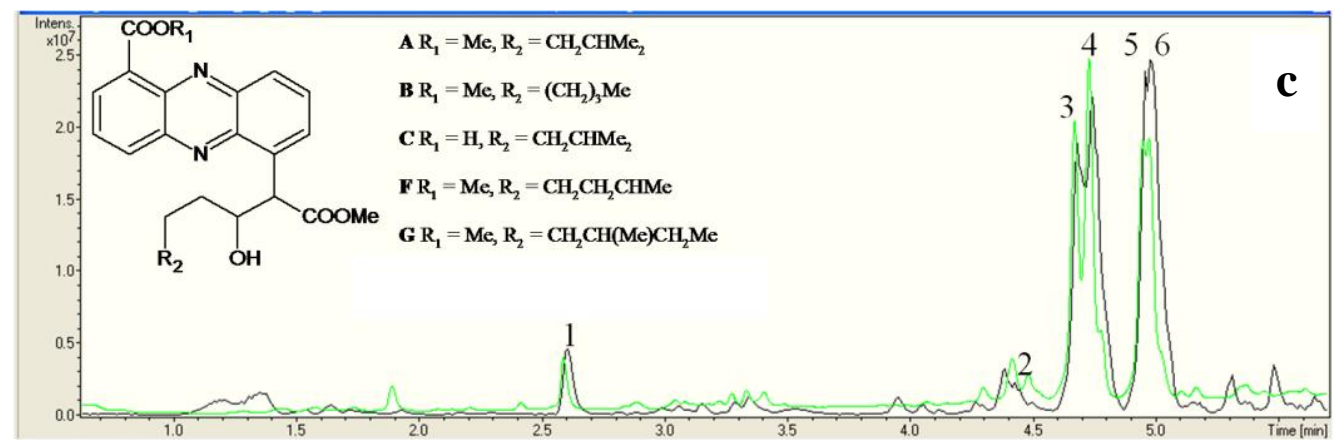
1) mayamycin
3) streptophenazine $A$ 5) streptophenazine $F$ $m / z 463$ $m / z 424$ $m / z 438$
2) streptophenazine $C$ $m / z 410$
4) streptophenazine $B$
6) streptophenazine $G$ $\mathrm{m} / z 424$ $m / z 438$ 
Figure 5. Structures of selected phenazines produced by Pseudomonas chlororaphis subsp. chlororaphis DSM 50083 ${ }^{\mathrm{T}}$ and Streptomyces cinnamonensis DSM $1042^{\mathrm{T}}$.<smiles>Oc1ccc2nc3ccccc3nc2c1</smiles>

2-Hydroxy-phenazine



Endophenazine A



Endophenazine B

In nine of the culture extracts a total of 13 different substances showed typical phenazine like UV-absorption spectra, but gave no hit in the databases concerning UV and mass data. This indicates the presence of unidentified and possibly new natural phenazine products which warrant further investigation.

For some of the identified phenazines interesting biological activities were reported. Senacarcin A is known for its activity against Gram-positive bacteria and tumor cell lines [45] and aestivophoenin C has antioxidative activity and acts as a neuronal cell protecting substance [44]. Interesting bioactivities of phenazines are also known from the marine Streptomyces sp. strain HB202, which produced several streptophenazines with activity against Gram-positive bacteria [39].

We expect that investigation of other so far unidentified phenazines from marine Actinobacteria is a remunerative challenge. Interestingly, phenazines were not detected in culture extracts of $p h z \mathrm{E}$ positive strains of Alphaproteobacteria and Firmicutes. Though, all bacteria containing a phzE phenazine gene fragment have the capability to synthesize the phenazine core structure, proof of gene fragments from a biosynthetic pathway does not give evidence of the integrity of corresponding gene cluster. Additionally, the expression of a gene cluster under conditions used is not warranted. Therefore, it is most likely that the cultivation conditions used were not appropriate for the production of some of the phenazines and have to be modified for the selected strains by our genetic approach in further studies.

\section{Experimental Section}

\subsection{Bacterial Strains and Their Phylogenetic Affiliation}

166 bacterial strains used in this study were of diverse phylogenetic affiliation and were isolated from Halichondria panicea (HB strains) [46] and Saccharina latissima (synonym Laminaria saccharina) (LB strains) [47] collected at the Kiel Fjord, Germany, and also from different sponges collected from the Adriatic Sea near Rovinj, Croatia (AB strains). The strains belong to six different phylogenetic groups (Table 1). Additionally, type strains known to produce phenazines were used as positive controls: Streptomyces cinnamonensis DSM $1042^{\mathrm{T}}$, Pseudomonas chlororaphis subsp. chlororaphis DSM 50083 $3^{\mathrm{T}}$, Pseudomonas chlororaphis subsp. aureofaciens DSM $6698^{\mathrm{T}}$, and Pseudomonas chlororaphis subsp. aurantiaca DSM $19603^{\mathrm{T}}$. For S. cinnamonensis DSM $1042^{\mathrm{T}}$ phenazine gene sequences and the production of different endophenazines and PCA (phenazine-1-carboxylic acid) have been demonstrated [15]. P. chlororaphis subsp. chlororaphis produced chlororaphin [48], P. chlororaphis 
subsp. aureofaciens and P. chlororaphis subsp. aurantiaca produces 2-hydroxy-phenazine [42] and phenazine-1-carboxylic acid [41], respectively.

For identification of the strains 16S rRNA gene sequence analyses were carried out according to Thiel et al. 2007 [49]. Comparison of the 16S rDNA sequences was performed using the EMBL nucleotide database available at the European Bioinformatics Institute homepage using the Basic Local Alignment Search Tool (nucleotide blast) [50] and the Ribosomal Database Project (RDP) database [51].

\subsection{Design of Oligonucleotides for Molecular Detection of phzE Phenazine Gene Fragments}

For the primer construction, amino acid sequences and nucleotide sequences of different $p h z \mathrm{E}$ genes were retrieved from the European Bioinformatics Institute homepage and aligned using the program CLUSTAL_X [52]. Nucleotide sequences were deduced from amino acid sequences. The alignment was analyzed manually. The following $p h z \mathrm{E}$ sequences were used for primer design: Streptomyces cinnamonensis (AM384985/CAL34110/68793...70757; putative 2-amino-2-desoxy-isochorismate synthase), Pseudomonas chlororaphis PCL1391 (AF195615/AAF17499/4873...6786; phenazine-1-carboxamide), Pseudomonas aeruginosa PAO1 (AF005404/AAC64488/3294...5177; pyocyanin), and Pseudomonas aeruginosa PAO1 (AE004091/AAG07601/4716660...4718543/ AAG05292/2073555...2075438; phenazine biosynthesis protein PhzE). Primers (Table 4) were synthesized by MWG (Ebersbach, Germany). In order to check the specificity of the primers, the sequences of $p h z \mathrm{Ef}$ and $p h z \mathrm{Er}$ were compared with sequences from the EMBL database using the Basic Local Alignment Search Tool (http://blast.ncbi.nlm.nih.gov/Blast.cgi). This comparison revealed $100 \%$ identity of the primers with corresponding sites of phenazine biosynthesis genes. Since Ashenafi et al. (2008) [53] reported that the anthranilate synthase (SvTrpEG) of Streptomyces venezuelae has a high degree of amino acid sequence similarity to the phenazine biosynthetic enzyme PhzE, the corresponding nucleotide sequence (AF01267) was compared with the $p h z \mathrm{E}$ primers using the bl2seq tool (http://blast.ncbi.nlm.nih.gov/Blast.cgi). No significant similarity was found indicating that false positive results are excluded.

Table 4. Primers used in this study.

\begin{tabular}{llll}
\hline Primer & Sequence & Function & Ref. \\
\hline 27f & 5'-GAGTTTGATCCTGGCTCAG-3' & PCR of the 16S rRNA gene & {$[54]$} \\
1492r & 5'-GGTTACCTTGTTACGACTT-3' & PCR of the 16S rRNA gene & {$[54]$} \\
534r & 5'-ATTACCGCGGCTGCTGG-3' & Sequencing of the 16S rRNA gene & {$[55]$} \\
342f & 5'-TACGGGAGGCAGCAG-3' & sequencing of the 16S rRNA gene & {$[55]$} \\
790f & 5'-GATACCCTGGTAGTCC-3' & sequencing of the 16S rRNA gene & {$[50]$} \\
$p h z$ Ef & 5'-GAAGGCGCCAACTTCGTYATCAA-3' & PCR and sequencing of $p h z$ E gene & this study \\
phzEr & 5'-GCCYTCGATGAAGTACTCGGTGTG-3' & PCR and sequencing of $p h z$ E gene & this study \\
Ps_up1 & 5'-ATCTTCACCCCGGTCAACG-3' & PCR and sequencing of $p h z$ F gene & {$[21]$} \\
Ps_low1 & 5'-CCRTAGGCCGGTGAGAAC-3' & PCR and sequencing of $p h z$ F gene & {$[21]$} \\
\hline
\end{tabular}




\subsection{Amplification and Identification of the Phenazine Gene Fragments}

The amplification reactions were carried out in a final volume of $25 \mu \mathrm{L}$. Taq DNA Polymerase (New England BioLabs, Ipswich, UK; MA, $5 \mathrm{U}_{\text {reaction }}{ }^{-1}$ ) with the ThermoPol Buffer Kit (New England BioLabs, Ipswich, UK; MA, USA) was applied. Primers $p h z E f$ and $p h z E r$ were deployed in a $10 \mu \mathrm{M}$ concentration. $1 \mu \mathrm{L}$ of a preparation containing each deoxynucleoside triphosphate at a concentration of $2.5 \mathrm{mM}$ was used. 10 to $50 \mathrm{ng}$ DNA of all strains used in this study was employed as template.

The amplification of the $p h z \mathrm{~F}$ gene sequence of the pseudomonads used in this study was performed using puReTaq Ready-To-Go polymerase chain reaction Beads (Amersham Biosciences, Uppsala, Sweden) with the primers Ps_up1 and Ps_low1 [21]. Cycler conditions for both PCR experiments were as follows: Initial denaturation: $94{ }^{\circ} \mathrm{C}$ for $120 \mathrm{~s}$ followed by 36 cycles of primer annealing at $54.7{ }^{\circ} \mathrm{C}$ (phzE) and $57^{\circ} \mathrm{C}(p h z \mathrm{~F})$, respectively, for $60 \mathrm{~s}$; primer extension at $72{ }^{\circ} \mathrm{C}$ for $120 \mathrm{~s}$ and denaturation at $94{ }^{\circ} \mathrm{C}$ for $60 \mathrm{~s}$. A final extension of $72{ }^{\circ} \mathrm{C}$ for $420 \mathrm{~s}$ was performed. All PCR reactions were conducted in a 11 thermocycler (Whatman Biometra ${ }^{\circledR}$, Göttingen, Germany). Results of the amplifications were checked on a $1.5 \%$ agarose gel stained with ethidium bromide. DNA sequencing was done according to Wiese et al. [47]. The comparison of the $p h z \mathrm{E}$ and $p h z \mathrm{~F}$ fragments, respectively, was done in the EMBL nucleotide database available at the European Bioinformatics Institute homepage using the Basic Local Alignment Search Tool (blastx) [50].

\subsection{Cultivation of phzE Strains}

\subsubsection{Cultivation of $p h z$ E Positive Strain}

All strains with a positive result for phenazine gene fragments were cultivated for subsequent chemical analysis of their cell extracts. They were grown on GYM agar plate $(4 \mathrm{~g}$ glucose, $4 \mathrm{~g}$ yeast extract, $4 \mathrm{~g}$ malt extract, $2 \mathrm{~g} \mathrm{CaCO}_{3}, 15 \mathrm{~g}$ agar, $1 \mathrm{~L}$ water, $\mathrm{pH}$ 7.2) or MB agar plate (37.4 g Difco marine broth, $15 \mathrm{~g}$ agar, $1 \mathrm{~L}$ water, $\mathrm{pH} 7.2$ ) at $28{ }^{\circ} \mathrm{C}$ for 17 days as well as in $100 \mathrm{~mL}$ and $1000 \mathrm{~mL}$ GYM (4 g glucose, $4 \mathrm{~g}$ yeast extract, $4 \mathrm{~g}$ malt extract, $1 \mathrm{~L}$ water, $\mathrm{pH}$ 7.2) or MB medium (37.4 g Difco marine broth, $1 \mathrm{~L}$ water, $\mathrm{pH} 7.2$ ) at $28{ }^{\circ} \mathrm{C}$ and $120 \mathrm{rpm}$ for seven days. The three P. chlororaphis strains were cultivated in $1000 \mathrm{~mL}$ King B medium [56] at $28^{\circ} \mathrm{C}$ and $120 \mathrm{rpm}$ for $24 \mathrm{~h}$.

\subsubsection{Cultivation of $p h z E$ Negative Strains}

All strains with a negative result for phenazine gene fragments were cultivated for subsequent chemical analysis of their cell extracts. The precultures were grown on TSB medium agar plates (tryptic soy broth[Difco], $12 \mathrm{~g} / \mathrm{L}$; $\mathrm{NaCl} 20 \mathrm{~g} / \mathrm{L}$; agar $15 \mathrm{~g} / \mathrm{L}$ ) at $28^{\circ} \mathrm{C}$ for 1 day. A $1 \mathrm{~cm}^{2}$ piece of the agar plate was used for inoculation of the main culture. Main cultures were grown in $100 \mathrm{~mL}$ TSB medium (with four baffles) and KingB medium [56] at $28^{\circ} \mathrm{C}$ and $120 \mathrm{rpm}$ for one, three and six days.

\subsection{Culture Extracts of phzE Positive and Negative Strains}

For $1 \mathrm{~L}$ cultures the supernatants were separated from the cell mass pellets by centrifugation at $4.700 \times \mathrm{g}$ for $20 \mathrm{~min}$ and extracted separately. Cells were homogenized by addition of $150 \mathrm{~mL} 96 \%$ 
EtOH and using Ultra-Turrax (IKA, Staufen, Germany) at 13,000 rpm for $30 \mathrm{~s}$. The extracts were dried in vacuo and redissolved in $\mathrm{MeOH}$ for further analyses. Supernatants and the other cultures were extracted with EtOAc by homogenization with the help of Ultra-Turrax at 16,000 rpm for $30 \mathrm{~s}$, also dried in vacuo and redissolved in $\mathrm{MeOH}$ for further analyses.

\subsection{Chemical Analysis of phzE Positive and Negative Strains}

Reversed phase HPLC experiments were performed using a $\mathrm{C}_{18}$ column (Phenomenex Onyx Monolithic $\mathrm{C} 18,100 \times 3.00 \mathrm{~mm}$ ) applying an $\mathrm{H}_{2} \mathrm{O}(\mathrm{A}) / \mathrm{MeCN}(\mathrm{B})$ gradient with $0.1 \% \mathrm{HCOOH}$ added to both solvents (gradient $0 \mathrm{~min} 5 \% \mathrm{~B}, 4 \mathrm{~min} 60 \% \mathrm{~B}, 6 \mathrm{~min} 100 \% \mathrm{~B}$; flow $2 \mathrm{~mL} / \mathrm{min}$ ) on a VWR Hitachi Elite LaChrom system coupled to an ESI-ion trap detector (Esquire 4000, Bruker Daltonics). Dereplication of substances was realized by comparison of MS and UV data obtained by HPLC-UV/MS analyses used data from the Antibase [57] and the Chapman \& Hall/CRC Dictionary of Natural Products databases [58]. For endophenazines $\mathrm{A}$ and B, 2-hydroxy-phenazine and phenazine-1-carboxylic acid structure was confirmed by ${ }^{1} \mathrm{H}$ NMR analysis.

\subsection{Nucleotide Sequence Accession Numbers}

The nucleotide sequence data reported in the present study were deposited in the GenBank nucleotide sequence database under the accession numbers HM460698 (AB049), HM460699 (AB108), AJ849545 (YIM 90018), AM231308 (YIM 36723), GQ863906 (HB117), GQ863907 (HB122), GQ863918 (HB202), GQ863921 (HB253), GQ863922 (HB254), GQ863926 (HB291), AM749667 (LB066), AM913982 (LB114), AM913952 (LB129), AM913970 (LB150) and AM913971 (LB151) for 16S rRNA and HM460700-HM460715 for phzE gene fragments.

\section{Conclusions}

In conclusion, the application of the phzE primer system is a useful tool to indicate the presence of the phenazine biosynthetic pathway in various groups of bacteria. The proof of concept was shown for well known producers of phenazines, but also for marine Streptomyces sp. strain. This approach is particularly relevant, because many marine Actinobacteria turned out to be active phenazine producers and Streptomyces strains are known to synthesize phenazines with anticancer and/or anti-infective activities [59]. The method used in this study offers a promising method to test the ability of producing phenazines in new isolates of all kinds of bacteria including marine Actinobacteria.

\section{Acknowledgements}

The authors gratefully thank B. Ohlendorf and N. Jansen for the NMR-analyses, A. Labes and I. Kajahn for fruitful discussions and K. Schumann for help with the cultivation experiments This study was supported by the Ministerium für Wissenschaft, Wirtschaft und Verkehr in the frame of the program "Zukunft Wirtschaft", which is co-financed by the European Union (EFRE). 


\section{References}

1. Blunt, J.W.; Copp, B.R.; Munro, M.H.G.; Northcote, P.T.; Prinsep, M.R. Marine natural products. Nat. Prod. Rep. 2011, 28, 196-268.

2. Newman, D.J.; Cragg, G.M. Natural products as sources of new drugs over the last 25 years. J. Nat. Prod. 2007, 70, 461-447.

3. Butler, M.S. Natural products to drugs: Natural product-derived compounds in clinical trials. Nat. Prod. Rep. 2008, 25, 475-516.

4. Donadio, S.; Monciardini, P; Sosio, M. Polyketide synthases and nonribosomal peptide synthetases: The emerging view from bacterial genomics. Nat. Prod. Rep. 2007, 24, 1073-1109.

5. Willey, J.M.; van der Donk, W.A. Lantibiotics: Peptides of diverse structure and function. Annu. Rev. Microbiol. 2007, 61, 477-501.

6. Arbiser, J.L.; Moschella, S.L. Clofazimine: A review of its medical uses and mechanisms of action. J. Am. Acad. Dermatol. 1995, 32, 241-247.

7. McDonald, M.; Mavrodi, D.V.; Thomashow, L.S.; Floss, H.G. Phenazine biosynthesis in Pseudomonas fluorescens: Branchpoint from the primary shikimate biosynthetic pathway and role of phenazine-1,6-dicarboxylic acid. J. Am. Chem. Soc. 2001, 123, 9459-9460.

8. Pierson III, L.S.; Gaffney, T.; Lam, S.; Gong, F. Molecular analysis of genes encoding phenazine biosynthesis in the biological control bacterium Pseudomonas aureofaciens 30-84. FEMS Microbiol. Lett. 1995, 134, 299-307.

9. Pusecker, K.; Laatsch, H.; Helmke, E.; Weyland, H. Dihydrophencomycin methyl ester, a new phenazine derivative from a marine Streptomycete. J. Antibiot. 1997, 50, 479-483.

10. Van Niekerk, S.; Huygens, F.; van Rensburg, C.E.J. A time-kill study to evaluate the in vitro activity of clofazimine in combination with cefotaxime against a penicillin- and cefotaxime-resistant strain of Streptococcus pneumoniae. J. Antimicrob. Chemother. 1997, 40, 602-604.

11. Van Rensburg, C.E.J.; Joone, G.K.; O’Sullivan, J.F.; Anderson, R.; Antimicrobial activities of clofazimine and B669 are mediated by lysophospholipids. Antimicrob. Agents Chemother. 1992, 36, 2729-2735.

12. Mavrodi, D.V.; Blankenfeldt, W.; Thomashow, L.S. Phenazine compounds in fluorescent Pseudomonas spp. biosynthesis and regulation. Annu. Rev. Phytopathol. 2006, 44, 417-445.

13. Reddy, V.M.; O'Sullivan, J.F.; Gangadharam, P.R. Antimycobacterial activities of riminophenazines. J. Antimicrob. Chemother. 1999, 43, 615-623.

14. Spicer, J.A.; Gamage, S.A.; Rewcastle, G.W.; Finlay, G.J.; Bridewell, D.J.; Baguley, B.C.; Denny, W.A. Bis(phenazine-1-carboxamides): Structure-activity relationships for a new class of dual topoisomerase I/II-directed anticancer drugs. J. Med. Chem. 2000, 43, 1350-1358.

15. Haagen, Y.; Glück, K.; Fay, K.; Kammerer, B.; Gust, B.; Heide, L. A gene cluster for prenylated naphthoquinone and prenylated phenazine biosynthesis in Streptomyces cinnamonensis DSM 1042. Chembiochem 2006, 7, 2016-2027.

16. Mavrodi, D.V.; Ksenzenko, V.N.; Bonsall, R.F.; Cook, R.J.; Boronin, A.M.; Thomashow, L.S. A seven-gene locus for synthesis of phenazine-1-carboxylic acid by Pseudomonas fluorescens 2-79. J. Bacteriol. 1998, 180, 2541-2548. 
17. Mentel, M.; Ahuja, E.G.; Mavrodi, D.V.; Breinbauer, R.; Thomashow, L.S.; Blankenfeldt, W. Of two make one: The biosynthesis of phenazines. Chembiochem 2009, 10, 2295-2304.

18. McDonald, M.; Mavrodi, D.V.; Thomashow, L.S.; Floss, H.G. Phenazine biosynthesis in Pseudomonas fluorescens: Branchpoint from the primary shikimate biosynthetic pathway and role of phenazine-1,6-dicarboxylic acid. J. Am. Chem. Soc. 2001, 123, 9459-9460.

19. Pierson III, L.S.; Pierson, E.A. Metabolism and function of phenazines in bacteria: Impacts on the behavior of bacteria in the environment and biotechnological processes. Appl. Microbiol. Biotech. 2010, 86, 1659-1670.

20. Calhoun, D.H.; Carson, M.; Jensen, R.A. The branch point metabolite for pyocyanine biosynthesis in Pseudomonas aeruginosa. J. Gen. Microbiol. 1972, 72, 581-583.

21. Mavrodi, D.V.; Peever, T.L.; Mavrodi, O.V.; Parejko, J.A.; Raaijmakers, J.M.; lemanceau, P.; Mazurier, S.; Heide, L.; Blankenfeldt, W.; Weller, D.M.; et al. Diversity and evolution of the phenazine biosynthesis pathway. Appl. Environ. Microbiol. 2010, 76, 866-879.

22. Fiedler, H.P.; Bruntner, C.; Bull, A.T.; Ward, A.C.; Goodfellow, M.; Potterat, O.; Puder, C.; Mihm, G. Marine actinomycetes as a source of novel secondary metabolites. Anton. Leeuwenhoek 2005, 87, 37-42.

23. Jensen, P.R.; Mincer, T.J.; Williams, P.G.; Fenical, W. Marine actinomycete diversity and natural product discovery. Anton. Leeuwenhoek 2005, 87, 43-48.

24. Salomon, C.E.; Magarvey, N.A.; Sherman, D.H. Merging the potential of microbial genetics with biological and chemical diversity: An even brighter future for marine natural product drug discovery. Nat. Prod. Rep. 2004, 21, 105-121.

25. Williams, P.G. Panning for chemical gold: Marine bacteria as a source of new therapeutics. Trends Biotech. 2009, 27, 45-52.

26. Gulder, T.A.M.; Moore, B.S. Chasing the treasures of the sea-Bacterial marine natural products. Curr. Opin. Microbiol. 2009, 12, 252-260.

27. Metsä-Ketelä, M.; Salo, V.; Halo, L.; Hautala, A.; Hakala, J.; Mäntsälä, P.; Ylihonko, K. An efficient approach for screening minimal PKS genes from Streptomyces. FEMS Microbiol. Lett. 1999, 180, 1-6.

28. Piel, J. A polyketide synthase-peptide synthetases gene cluster from an uncultured bacterial symbiont of Paederus beetles. Proc. Natl. Acad. Sci. USA 2002, 99, 14002-14007.

29. Laursen, J.B.; de Visser, P.C.; Nielsen, H.K.; Jensen, K.J.; Nielsen, J. Solid-phase synthesis of new saphenamycin analogues with antimicrobial activity. Bioorg. Med. Chem. Lett. 2002, 12, 171-175.

30. Price-Whelan, A.; Dietrich, L.E.; Newman, D.K. Rethinking 'secondary' metabolism: Physiological roles for phenazine antibiotics. Nat. Chem. Biol. 2006, 2, 71-78.

31. Thomashow, L.S.; Weller, D.M.; Bonsall, R.F.; Pierson, L.S. Production of the antibiotic phenazine-1-carboxylic acid by fluorescent Pseudomonas species in the rhizosphere of wheat. Appl. Environ. Microbiol. 1990, 56, 908-912.

32. Wilson, R.; Sykes, D.A.; Watson, D.; Rutman, A.; Taylor, G.W.; Cole, P.J. Measurement of Pseudomonas aeruginosa phenazine pigments in sputum and assessment of their contribution to sputum sol toxicity for respiratory epithelium. Infect. Immun. 1988, 56, 2515-2517. 
33. Angell, S.; Bench, B.J.; Williams, H.; Watanabe, C.M.H. Pyocyanin isolated from a marine microbial population: Synergistic production between two distinct bacterial species and mode of action. Chem. Biol. 2006, 13, 1349-1359.

34. Isnansetyo, A.; Kamei, Y. Bioactive substances produced by marine isolates of Pseudomonas. J. Ind. Microbiol. Biotech. 2009, 36, 1239-1248.

35. Jayatilake, G.S.; Thornton, M.P.; Leonard, A.C.; Grimwade, J.E.; Baker, B.J. Metabolites from an Antarctic sponge-associated bacterium, Pseudomonas aeruginosa. J. Nat. Prod. 1996, 59, 293-296.

36. Imamura, N.; Nishijima, M.; Takadera, T.; Adachi, K.; Sakai, M.; Sano, H. New anticancer antibiotics pelagiomicins, produced by a new marine bacterium Pelagiobacter variabilis. J. Antibiot. 1997, 50, 8-12.

37. Choi, E.J.; Kwon, H.C.; Ham, J.; Yang, H.O. 6-Hydroxymethyl-1-phenazine-carboxamide and 1,6-phenazinedimethanol from a marine bacterium, Brevibacterium sp. KMD 003, associated with marine purple vase sponge. J. Antibiot. 2009, 62, 621-624.

38. Li, D.; Wang, F.; Xiao, X.; Zeng, X.; Gu, Q.Q.; Zhu, W. A new cytotoxic phenazine derivative from a deep sea bacterium Bacillus sp. Arch. Pharm. Res. 2007, 30, 552-555.

39. Mitova, M.I.; Lang, G.; Wiese, J.; Imhoff, J.F. Subinhibitory concentrations of antibiotics induce phenazine production in a marine Streptomyces sp. J. Nat. Prod. 2008, 71, 824-827.

40. Turner, J.M.; Messenger, A.J. Occurrence, biochemistry and physiology of phenazine pigment production. Adv. Microb. Physiol. 1986, 27, 211-275.

41. Brisbane, P.G.; Janik, L.J.; Tate, M.E.; Warren, R.F. Revised structure for the phenazine antibiotic from Pseudomonas fluorescens 2-79 (NRRL B-15132). Antimicrob. Agents Chemother. 1987, 31, 1967-1971.

42. Levitch, M.E.; Rietz, P. The isolation and characterization of 2-hydroxyphenazine from Pseudomonas aureofaciens. Biochemistry 1966, 5, 689-692.

43. Kanner, D.; Gerber, N.N.; Bartha, R. Pattern of phenazine pigment production by a strain of Pseudomonas aeruginosa. J. Bacteriol. 1978, 134, 690-692.

44. Kunigami, T.; Shin-Ya, K.; Furihata, K.; Furihata, K.; Hayakawa, Y.; Seto, H. A novel neuronal cell protecting substance, aestivophoenin C, produced by Streptomyces purpeofuscus. J. Antibiot. 1998, 51, 880-882.

45. Nakano, H.; Yoshida, M.; Shirahata, K.; Ishii, S.; Arai, Y.; Morimoto, M.; Tomita, F. Senacarcin A, a new antitumor antibiotic produced by Streptomyces endus subsp. aureus. J. Antibiot. 1982, 35, 760-762.

46. Schneemann, I.; Nagel, K.; Kajahn, I.; Labes, A.; Wiese, J.; Imhoff, J.F. Comprehensive investigations of marine Actinobacteria associated with the sponge Halichondria panicea. Appl. Environ. Microbiol. 2010, 76, 3702-3714.

47. Wiese, J.; Thiel, V.; Nagel, K.; Staufenberger, T.; Imhoff, J.F. Diversity of antibiotic-active bacteria associated with the brown alga Laminaria saccharina from the Baltic Sea. Mar. Biotech. 2009, 11, 287-300.

48. Peix, A.; Valverde, A.; Rivas, R.; Igual, J.M.; Ramírez-Bahena, M.H.; Mateos, P.F.; Santa-Regina, I.; Rodríguez-Barrueco, C.; Martínez-Molina, E.; Velázquez, E. Reclassification of Pseudomonas aurantiaca as a synonym of Pseudomonas chlororaphis and proposal of three 
subspecies, $P$. chlororaphis subsp. chlororaphis subsp. nov., $P$. chlororaphis subsp. aureofaciens subsp. nov., comb. nov. and $P$. chlororaphis subsp. aurantiaca subsp. nov., comb. nov. Int. J. Syst. Evol. Microbiol. 2007, 57, 1286-1290.

49. Thiel, V.; Neulinger, S.C.; Staufenberger, T.; Schmaljohann, R.; Imhoff, J.F. Spatial distribution of sponge-associated bacteria in the Mediterranean sponge Tethya aurantium. FEMS Microbiol. Ecol. 2007, 59, 47-63.

50. Altschul, S.F.; Gish, W.; Miller, W.; Myers, E.W.; Lipman, D.J. Basic Local alignment search tool. J. Mol. Biol. 1990, 215, 403-410.

51. Cole, J.R.; Wang, Q.; Cardenas, E.; Fish, J.; Chai, B.; Farris, R.J.; Klam-Syed-Mohideen, A.S.; McCarell, D.M.; Marsh, T.; Garrity, G.M.; et al. The Ribosoaml Database Project: Improved alignments and new tools for rRNA analysis. Nucl. Acids Res. 2009, 37, D141-D145.

52. Thompson, J.D.; Gibson, T.J.; Plewniak, F.; Jeanmougin, F.; Higgins, D.G. The CLUSTAL_X windows interface: Flexible strategies for multiple sequence alignment aided by quality tools. Nucl. Acid Res. 1997, 25, 4876-4882.

53. Ashenafi, M.; Carrington, R.; Collins, A.C.; Byrnes, W.M. The fused TrpEG from Streptomyces venezuelae is an anthranilate synthase, not an 2-amino-4-deoxyisochorismate (ADIC) synthase. Ethn. Dis. 2008, 18 (Suppl. 2), S2-9-13.

54. Weisburg, W.G.; Barns, S.M.; Pelletier, D.A.; Lane, D.J. 16S ribosomal DNA amplification for phylogenetic study. J. Bacteriol. 1991, 173, 697-703.

55. Muyzer, G.; de Waal, E.C.; Uitterlinden, A.G. Profiling of complex microbial populations by denaturing gradient gel electrophoresis analysis of polymerase chain reaction-amplified genes coding for 16S rRNA. J. Mol. Biol. 1993, 59, 695-700.

56. King, E.O.; Ward, M.; Raney, D.E. Two simple media for the demonstration of pyocyanin and fluorescein. J. Lab. Clin. Med. 1954, 44, 301-307.

57. Laatsch, H. Antibase 2007 SciDex: The Natural Products Identifier, 1st ed.; Wiley-VCH: Weinheim, Germany, 2007.

58. Buckingham, J. Dictionary of Natural Products on CD-ROM, 16.2 ed.; Chapman and Hall: London, UK, 2008.

59. Laursen, J.B.; Nielsen, J. Phenazine natural products: Biosynthesis, synthetic analogues, and biological activity. Chem. Rev. 2004, 104, 1663-1686.

Samples Availability: Available from the authors.

(C) 2011 by the authors; licensee MDPI, Basel, Switzerland. This article is an open access article distributed under the terms and conditions of the Creative Commons Attribution license (http://creativecommons.org/licenses/by/3.0/). 